\section{Gastric outlet obstruction syndrome due to an obstructed hepaticojejunostomy loop treated by one-step endoscopic ultrasound-guided gastro- enterostomy}
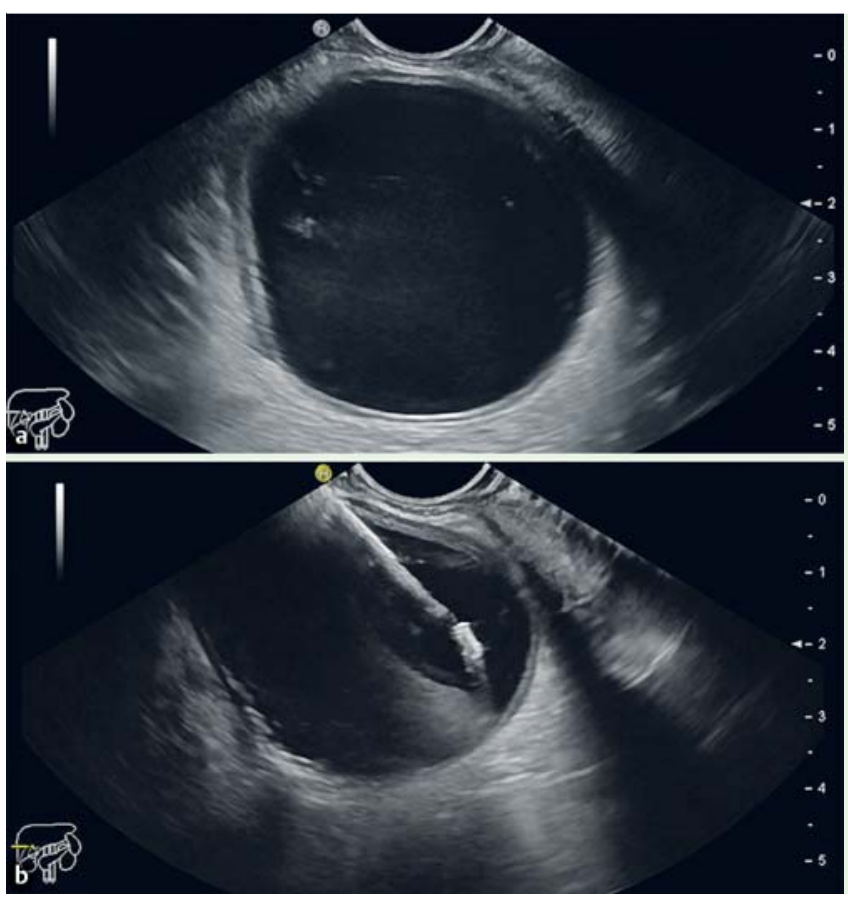

Fig. 1 Endoscopic ultrasound (EUS)guided gastroenterostomy to treat a patient with malignant obstruction of the afferent loop of a Roux-en-Y anastomosis. a Visualization of the dilated jejunal loop with EUS. b EUS-guided puncture of the jejunum using the Hot Axios stent delivery system.
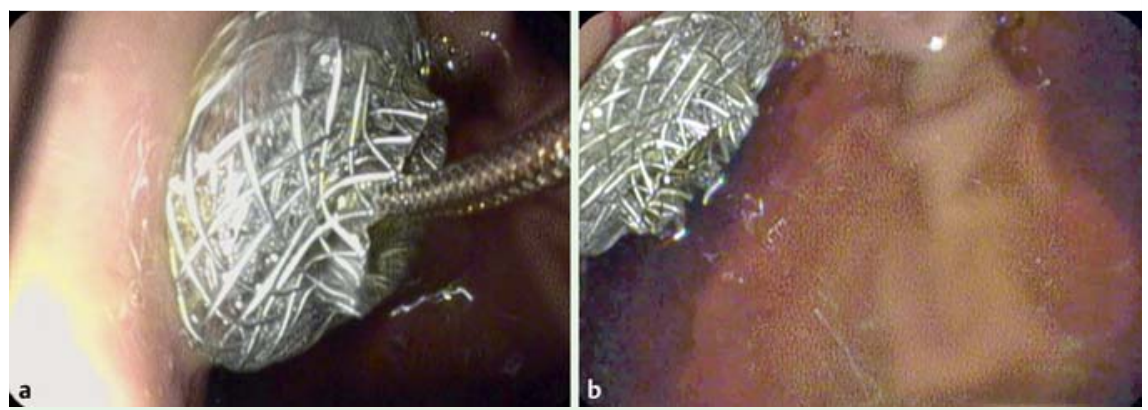

Fig. 2 a The lumen-apposing stent with the delivery system in the stomach. $\mathbf{b}$ The stent is fully deployed.

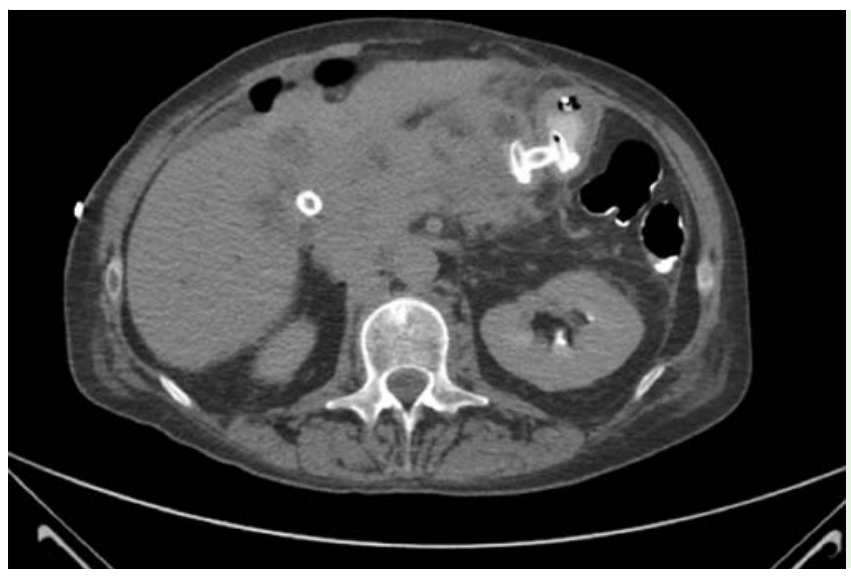

Endoscopic ultrasound (EUS)-guided gastroenterostomy is an experimental procedure that is emerging as a minimally invasive alternative to surgical bypass and to luminal endoscopic stent placement $[1,2]$. We report successful treatment of a 50 -year-old woman who was referred with malignant obstruction of the afferent loop of a Roux-en-Y anastomosis due to local recurrence of cholangiocarcinoma following an earlier pancreaticoduodenectomy. Because the severely dilated afferent loop was compressing the stomach, sufficient nutrition was impossible. Additionally, the patient was jaundiced because of extrahepatic biliary obstruction.

A percutaneous transhepatic metal stent was inserted to relieve the jaundice, but after several unsuccessful attempts to place self-expandable stents through the afferent loop stenosis, we decided to perform an EUS-guided gastroenterostomy using a linear-scanning echoendoscope (Pentax EG-3870UTK; Tokyo, Japan) and the Hot Axios stent system (Hot Axios; Xlumena, Mountain View, California, USA). After the dilated jejunal loop was visualized by EUS, the distal end of the stent delivery system was inserted through the stomach wall into the jejunal lumen by applying electrocautery ( $\bullet$ Fig.1a,b, Video 1). The lumen-apposing stent was then deployed resulting in immediate flow of fluid from the obstructed jejunal loop into the stomach. Fluoroscopy was not performed since correct placement could be confirmed by EUS and direct view of the jejunal lumen ( $\bullet$ Fig.2a,b, $\bullet$ Video 2). Following the procedure, the patient's symptoms resolved and enteral feeding was reinstated ( Fig.3). No adverse events were observed during a 30-day follow-up period.

Currently, two case series and several case reports have been published describing different methods such as the double-balloon technique and water infusion [2-5]. In patients with afferent loop obstruction, direct EUS-guided gastroenterostomy without the use of fluoroscopy or other adjuncts can safely be performed since the dilated jejunal loop is filled with fluids, but further research is warranted before large-scale clinical implementation.

Endoscopy_UCTN_Code_TTT_1AS_2AG

Competing interests: None 


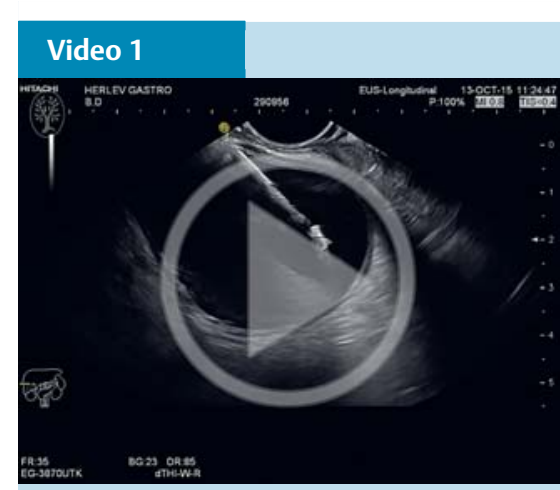

Endoscopic ultrasound (EUS)-guided gastroenterostomy: puncture of the jejunum.

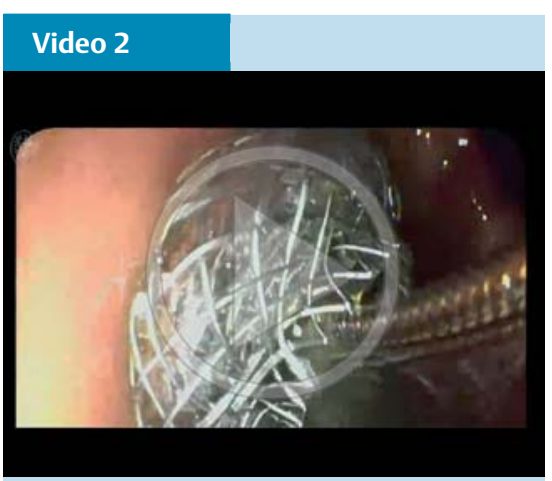

Deployment of the lumen-apposing stent as seen with gastroscopy. Fluids are flowing from the jejunal loop into the stomach.
Bojan Kovacevic ${ }^{1}$, Peter Vilmann', John G. Karstensen ${ }^{1,2}$

${ }^{1}$ Gastro Unit, Division of Endoscopy, Department of Gastroenterology, Copenhagen University Hospital Herlev, Denmark

${ }^{2}$ Gastro Unit, Division of Surgery, Copenhagen University Hospital Hvidovre, Denmark

\section{References}

1 Nagaraja V, Eslick GD, Cox MR. Endoscopic stenting versus operative gastrojejunostomy for malignant gastric outlet obstruction - a systematic review and meta-analysis of randomized and non-randomized trials. J Gastrointest Oncol 2014; 5: 92 -98

2 Itoi T, Ishii $K$, Ikeuchi $N$ et al. Prospective evaluation of endoscopic ultrasonography-guided double-balloon-occluded gastrojejunostomy bypass (EPASS) for malignant gastric outlet obstruction. Gut 2016; 65: 1931955. DOI: 10.1136/gutjnl-2015310348. Epub 2015 Aug 17

3 Khashab MA, Kumbhari V, Grimm IS et al. EUS-guided gastroenterostomy: the first $U$. S. clinical experience (with video). Gastrointest Endosc 2015; 82: 932 - 938

4 Taunk P, Cosgrove N, Loren DE et al. Endoscopic ultrasound-guided gastroenterostomy using a lumen-apposing self-expanding metal stent for decompression of afferent loop obstruction. Endoscopy 2015; 47: E395-E396. DOI: 10.1055/s-0034-1392564

5 Chatterjee S, Ibrahim B, Charnley RM et al. Endoscopic ultrasound-guided gastroenterostomy for palliative drainage of an obstructed hepaticojejunostomy loop. Endoscopy 2011; 43 (Suppl. 02): E1-E2. DOI: $10.1055 / \mathrm{s}-0030-1255720$
Bibliography

DOI http://dx.doi.org/

$10.1055 / \mathrm{s}-0042-102451$

Endoscopy 2016; 48: E103-E104

(c) Georg Thieme Verlag KG

Stuttgart · New York

ISSN 0013-726X

Corresponding author

Bojan Kovacevic, MD

Gastro Unit, Division of Endoscopy

Department of Gastroenterology

Copenhagen University Hospital Herlev

Herlev Ringvej 75

2730 Herlev

Denmark

Fax: +45-38-684009

bojan.kovacevic.02@regionh.dk 